\title{
Does Restraint Use Depend on the Hospital? A Multilevel Analysis of Multicentre Prevalence Measurements
}

Silvia Thomann ( $\sim$ silvia.thomann@bfh.ch )

Bern University of Applied Sciences

\section{Sabine Hahn}

Bern University of Applied Sciences

\section{Silvia Bauer}

Medical University of Graz

Dirk Richter

Bern University of Applied Sciences

\section{Sandra Zwakhalen}

Maastricht University

\section{Research Article}

Keywords: Hospitals, Multilevel Analysis, Organisational Culture, Quality of Health Care, Restraint

Posted Date: February 1st, 2021

DOI: https://doi.org/10.21203/rs.3.rs-146567/v1

License: (c) This work is licensed under a Creative Commons Attribution 4.0 International License. Read Full License

Version of Record: A version of this preprint was published at BMC Health Services Research on April 20th, 2021. See the published version at https://doi.org/10.1186/s12913-021-06362-y. 


\section{Abstract \\ Background}

In restraint use in the somatic acute-care hospital setting, routine and institutional culture seem to play an important role. This implies that similar patient situations would be managed with restraints in one hospital, while in another hospital the situation would be managed without restraints. This practice variation appears to be ethically and legally questionable. The influence of organisation-specific factors such as the availability of guidelines is discussed. However, the relevance of such factors at the hospital level has been rarely investigated to date. Therefore, the aims of this study were a) to determine how much variance in restraint use can be explained on the hospital level and b) to examine the impact of organisational factors on restraint use.

\section{Methods}

A secondary data analysis of cross-sectional multicentre data was performed. Data were collected during three quality measurements (2016-2018) in acute-care hospitals in Switzerland and Austria. Hospitalised patients from different medical specialties aged $18+$ with informed consent were included. Descriptive analysis and multilevel logistic regression analysis were performed.

\section{Results}

The study included 29,477 patients from a total of 140 hospitals. The 30-day prevalence rate of patients with at least one restraint was $8.7 \%(n=2,577)$. The availability of guidelines regarding restraint use and refresher courses for nursing staff were associated with less restraint use (odds ratios $=0.60$ and 0.76 ). By adding the hospital as a random effect, the explained variance of the model increased from $24-55 \%$.

\section{Conclusions}

The use of restraints varies widely between hospitals, even considering patient characteristics. The identification of situations in which restraints were used out of routine or institutional culture appears to be an important approach in restraint reduction. Investments in appropriate structures and employee knowledge can facilitate providing restraint-free care as much as possible.

\section{Background}

Restraint use in health care often leads to negative effects for patient health, such as functional decline, higher mortality, distress or trauma (1-4), and to moral distress for health professionals $(5,6)$. Therefore, a reduction in restraint use is recommended (7-9).

To date, quality improvement initiatives regarding restraint use are mainly known in the long-term care and mental health setting $(10,11)$. Nevertheless, restraints are frequently used in the somatic acute care hospital setting (henceforth referred to as 'hospital') as well. Prevalence rates up to $100 \%$ are reported $(1,12,13)$. Large differences in restraint prevalence rates can be detected depending on the ward type studied (intensive care units often have a much higher prevalence rate) and by the definition of restraints used (e.g. only restraint belts; alternatively, bed rails and electronic monitoring can also be considered as restraints). 
Frequently stated reasons for restraint use in the hospital setting are patient safety (e.g. fall prevention or prevention of therapy interruption) and patient characteristics like cognitive impairment $(5,14,15)$. However, evidence for the effectiveness of restraints for these reasons is lacking $(5,16,17)$. Nevertheless, restraints also seem to be used out of routine according to the tradition on the ward or local habits (18-21). This implies that practice variation may exist. Consequently, in a similar patient situation, restraints may be used in one hospital, while in another hospital this situation would be managed without restraints. These differences in restraint use among hospitals independent of evidence or professional recommendations appear to be ethically and legally questionable. In this context, the relevance and role of organisational factors such as structures, policies/guidelines, education for staff, monitoring of restraint use and organisational attitudes are discussed $(18,20-24)$.

Surprisingly, the practice variation in restraint use among hospitals and the impact of organisational factors has rarely been investigated to date. Nevertheless, in order to promote a professional management of restraints and, thus, to develop and implement effective measures for restraint reduction, it is crucial to know the influencing factors on different levels and their impact on the use and non-use of restraints. Therefore, the aims of this study were a) to determine how much variance in restraint use can be explained on the hospital level (hospital effect) and b) to examine the impact of organisational factors on restraint use; both aspects considered the influence of patient characteristics on restraint use.

\section{Methods}

\section{Study design and setting}

A secondary data analysis of cross-sectional multicentre studies was performed. Data were collected within the International Prevalence Measurement of Quality of Care, called LPZ (Landelijke Prevalentiemeting Zorgkwaliteit) International $(25,26)$. LPZ International performs an annual international quality measurement for a variety of care indicators (like pressure ulcers, falls and restraints) in various settings and countries. Healthcare institutions are invited annually by a national coordinator in several countries to participate on a voluntary basis in the measurement. For the present study, data from the hospital setting of Switzerland and Austria from three one-day measurement points in the years 2016 to 2018 were included. Other countries in the LPZ consortium were not able to provide data as very few hospitals measured restraint use.

\section{Sample}

Hospitalised patients from different medical specialties (ward types) aged $18+$ with informed verbal (Switzerland) or written (Austria) consent were included. Patients were excluded if they were not available on the ward during the measurement (e.g. since they were undergoing surgery) or could not give informed consent (e.g. due to cognitive impairment or language barriers) and where no legal representative was available.

\section{Instrument and data collection}

For data collection, the LPZ 2.0 instrument was used. It is the 2016 revised version of the LPZ instrument (25). With LPZ 2.0, general and care indicator specific information is assessed on the institutional, ward and patient levels. For this secondary data analysis, information regarding restraints of different levels was included (for details, see Table 1). Restraints were defined as 'interventions that may infringe [on] a person's human rights and freedom of movement, including observation, seclusion, manual restraint, mechanical restraint and rapid tranquillisation' (27). 


\begin{tabular}{|c|c|}
\hline Level & Information \\
\hline National & Country (Switzerland, Austria) \\
\hline \multirow[t]{2}{*}{ Institutional } & $\begin{array}{l}\text { Availability of a protocol/guidelines regarding restraints (based on a(n) (inter)national guideline) within } \\
\text { the institution (yes, no) }\end{array}$ \\
\hline & Availability of a multi-disciplinary expert committee regrading restraints within the institution (yes, no) \\
\hline \multirow[t]{2}{*}{ Ward } & $\begin{array}{l}\text { Regular audits are performed on the ward level to ensure compliance with the protocol/guidelines } \\
\text { regarding restraints (yes, no) }\end{array}$ \\
\hline & Refresher course regarding restraints for at least $80 \%$ of ward nursing staff in the last two years (yes, no) \\
\hline \multirow[t]{7}{*}{ Patient } & Age in years (interval) \\
\hline & Female gender (yes, no) \\
\hline & Surgical intervention in the two weeks prior to data collection (yes, no) \\
\hline & Number of days since admission to hospital (interval) \\
\hline & $\begin{array}{l}\text { Medical diagnosis groups according to International Statistical Classification of Diseases and Related } \\
\text { Health Problems 10th Revision (ICD-10; for each diagnosis group yes, no) }(28)\end{array}$ \\
\hline & $\begin{array}{l}\text { Care dependency assessed with the Care Dependency Scale (CDS) ( } 15 \text { items [e.g., eating and drinking or } \\
\text { mobility] are rated on a Likert scale from } 1 \text { to } 5 \text { [sum score } 15-75 \text {. Lower scores indicate higher care } \\
\text { dependency resulting in five verified categories: } 15-24 \text { completely dependent, } 25-44 \text { dependent to a } \\
\text { great extent, } 45-59 \text { partially dependent, } 60-69 \text { independent to a great extent, } 70-75 \text { completely } \\
\text { independent) (29) }\end{array}$ \\
\hline & Restraint use within the institution retrospectively over a maximum period of 30 days (yes, no) \\
\hline
\end{tabular}

Within LPZ 2.0, the data collection process is highly standardised. The whole process (e.g. recruitment and information of patients, preparing data collection including documentation of restraint use 30 days prior to data collection) and all questions and answer options are internationally defined and described in a measurement manual. Additionally, the questionnaire was conceived as an online data entry program leading the questionnaire completion. To ensure uniform execution of the measurement and uniform answering of the questions, data collectors were trained. Using the train-thetrainer procedure, the national coordinator trained the responsible person within each hospital (called the institutional coordinator). The institutional coordinator then trained the data collectors (registered nurses) within the hospital.

Additionally, the measurement manual with all the information was made available for the data collectors directly in the data entry program.

On the predetermined measurement day, the patient level data were collected by the trained data collectors on-site at the patient's bedside and/or through patient documentation (retrospective assessment). The questions on the institutional and ward level were answered by the institutional coordinator.

\section{Statistical analysis}

The data from the different measurement points and the two countries (Switzerland and Austria) were pooled into one dataset. Descriptive statistics (numbers, percentages, 95\% confidence intervals [CI], median, interquartile range [IQR]) were used to describe the organisational factors, the sample and the restraint prevalence rate.

A multilevel modelling approach was used in order to determine how much variance in restraint use can be explained on the hospital level (hospital effect). This means that the analysis took into account that patients are clustered in hospitals with their organisational factors. The baseline (before variable selection) of the multilevel logistic regression model was built as shown in Fig. 1. 
For variable selection, we used the Akaike information criterion (AIC) (30) backwards procedure implemented in the $\mathrm{R}$ package MASS (31). Here, however, the hospital random effect had to be treated as a fixed effect. During development of the analysis, we also considered using similar variable selection procedures for logistic multilevel models, but the few software implementations we found were not practicable for our problem. Since the hospital effect is an explicit part of the question, the AIC procedure was employed such that the hospital variable cannot be unselected. Further, to enhance the stability of the variable selection, i.e. to reduce the number of noisy variables selected due to the large sample size, we used a split-half approach where the AIC procedure was applied on both of two subsets from a random split of the data, and then only the variables included in both selections were used for the final multilevel model. The model then was built as a generalised linear mixed model fit by maximum likelihood (Laplace approximation) implemented in the R package Ime4 (32). Additionally, the ICC (intraclass correlation coefficient) was estimated, and a log-likelihood ratio test was performed to evaluate the relevance of the random effect.

Two ICD-10 diagnosis groups (congenital malformations, deformations and chromosomal abnormalities; certain conditions originating in the perinatal period) and the answer option unknown/no diagnosis were present in less than $1 \%$ of patients and would have led to convergence problems of the regression model. Therefore, these variables had to be excluded. For similar reasons, the variable age had to be centred. Multicollinearity was tested using the variance inflation factor (VIF). There were no missing data as the online data collection program only allowed for finishing the survey when all questions were answered.

The statistical analysis was conducted utilising R Version 4.0.1 (33) and the R Packages compareGroups (34), Hmisc (35), Ime4 (32), jtools (36), MASS (31), MuMIn (37), sjPlot (38), tableone (39) and tidyverse (40). For data cleaning and pooling, SPSS version 25 was used (41).

\section{Ethical considerations}

In Switzerland, the Ethics Committee of the Canton of Bern declared that the present study is not subject to the Swiss Human Research Act and ethical approval was not required (April 2019, BASEC-Nr: Req-2019-00259). In Austria, the Ethics Committee of the Medical University of Graz approved the study protocol (approval nr. 20-192 ex08/09). All patients or their legal representatives received written information about the measurement and gave their verbal (Switzerland) or written (Austria) informed consent. Data were collected pseudonymously so that no conclusions can be made regarding the individual patients. Participation was voluntary.

\section{Results}

The study included 29,477 patients from a total of 140 hospitals (Table 2). Of these, 20,561 (69.8\%) patients were assessed in Switzerland and 8,916 (30.2\%) patients in Austria. The 30-day prevalence rate of patients with at least one restraint was $8.7 \%(n=2,577)$. Differences between countries were evident. In Switzerland, the prevalence rate was much higher $(10.6 \%, n$ $=2,171)$ than in Austria $(4.6 \%, n=406)$. A more refined description about the differences between Switzerland and Austria as well as about the restraint type used, reasons for restraint use and process indicators is available in a publication by Thomann et al.(15)

Overall, $73.6 \%(n=21,694)$ of all patients were treated in a hospital with guidelines regarding restraints. A multi-disciplinary expert committee regarding restraints was implemented in the hospitals of $42.7 \%(n=12,575)$ of all patients assessed. On the ward level, regular audits to ensure compliance with the guidelines regarding restraints was performed in $68.3 \%(20,126)$ of all patients surveyed. In $21.1 \%(n=6,209)$ of all patient situations, nursing staff attended a refresher course on restraints. 


\begin{tabular}{|c|c|c|c|}
\hline \multirow{2}{*}{\multicolumn{2}{|c|}{$\begin{array}{l}\text { Characteristics } \\
\text { National level }\end{array}$}} & \multicolumn{2}{|c|}{ Total $(n=29,477)$} \\
\hline & & $\mathbf{n}$ & $\%(95 \% \mathrm{Cl})$ \\
\hline \multirow[t]{2}{*}{ Country } & Switzerland & 20,561 & $69.8(69.2-70.3)$ \\
\hline & Austria & 8,916 & $30.2(29.7-30.8)$ \\
\hline \multicolumn{4}{|c|}{ Institutional level } \\
\hline \multicolumn{2}{|c|}{ Guidelines regarding restraint use (yes) } & 21,694 & $73.6(73.1-74.1)$ \\
\hline \multicolumn{2}{|c|}{ Multi-disciplinary expert committee (yes) } & 12,575 & $42.7(42.1-43.2)$ \\
\hline \multicolumn{4}{|c|}{ Ward level } \\
\hline \multicolumn{2}{|c|}{ Regular audits (yes) } & 20,126 & $68.3(67.7-68.8)$ \\
\hline \multicolumn{2}{|c|}{ Refresher course (yes) } & 6209 & $21.1(20.6-21.5)$ \\
\hline \multicolumn{2}{|c|}{ Patient level } & median & IQR \\
\hline \multicolumn{2}{|c|}{ Age in years } & 70 & 24 \\
\hline \multicolumn{2}{|c|}{ Number of days since admission to hospital } & 5 & 9 \\
\hline \multirow{2}{*}{\multicolumn{2}{|c|}{ Care Dependency Scale (sum score) }} & 71 & 15 \\
\hline & & $\mathbf{n}$ & $\%(95 \% \mathrm{Cl})$ \\
\hline \multicolumn{2}{|c|}{ Female gender } & 14,504 & $49.2(48.6-49.8)$ \\
\hline \multicolumn{2}{|c|}{ Surgical intervention in the two weeks prior to data collection (yes) } & 10,542 & $35.8(35.2-36.3)$ \\
\hline \multicolumn{4}{|c|}{ Three most frequent ICD-10 diagnosis groups (multiple responses) } \\
\hline \multicolumn{2}{|c|}{ Diseases of the circulatory system } & 16,245 & $55.1(54.5-55.7)$ \\
\hline \multicolumn{2}{|c|}{ Endocrine, nutritional and metabolic diseases } & 9,886 & $33.5(33.0-34.1)$ \\
\hline \multicolumn{2}{|c|}{ Diseases of the musculoskeletal system and connective tissue } & 9,834 & $33.4(32.8-33.9)$ \\
\hline \multicolumn{2}{|c|}{ Restraint (yes) } & 2,577 & $8.7(8.4-9.1)$ \\
\hline
\end{tabular}

Based on the multilevel regression analysis, several factors associated with restraint use were found (Table 3). The strongest association was found for patients' care dependency: completely dependent patients in comparison to completely independent patients had an almost 40 times higher risk of being restrained (odds ratio [OR] 39.65, confidence interval [Cl] 32.66-48.13). A strong association was also found for patients with mental and behavioural disorders: the risk for them to be restrained was more than two times higher than for patients without such disorders (OR 2.31, $\mathrm{Cl} 2.09-2.56$ ).

With regard to the organisational factors, the availability of guidelines regarding restraints $(\mathrm{OR} 0.60, \mathrm{Cl} 0.49-0.74)$ and refresher courses for at least $80 \%$ of ward nursing staff (OR $0.76, \mathrm{Cl} 0.65-0.90)$ were associated with less restraint use. The availability of a multi-disciplinary expert committee and regular audits to ensure compliance with the protocol/guidelines regarding restraints were not selected for the model, indicating that these factors are not relevant concerning restraint use, from a statistical point of view. Also, the variable country was not selected for the model, despite large descriptive differences in prevalence rates. 
Only considering the fixed effects (patient characteristics and organisational factors), the model could explain $24 \%$ of the variance in restraint use (marginal $\mathrm{R}^{2}=0.24$ ). By adding the random effect (hospital as cluster variable), the model explains $55 \%$ of the variance in restraint use (conditional $\mathrm{R}^{2}=0.55$ ). The log-likelihood ratio test was statistically significant $(p$-value $<0.000$ ), indicating that adding hospital as a random effect (cluster) does improve the model. Additionally, the ICC (0.41) shows that the random effect is also relevant from a clinical point of view. This means that a relevant part of the variance in restraint use can be explained at the hospital level.

Table 3

Multilevel logistic regression model

\begin{tabular}{|c|c|}
\hline \multicolumn{2}{|l|}{$\begin{array}{l}\text { Model: AIC 12590.5; marginal } \mathrm{R}^{2}=0.24 \text {; } \\
\text { conditional } \mathrm{R}^{2}=0.55 ; \mathrm{ICC}=0.41\end{array}$} \\
\hline Random effect & Variance (SD) \\
\hline Hospital (intercept) & $2.28(1.51)$ \\
\hline Fixed effects & OR $(95 \% \mathrm{Cl})$ \\
\hline (intercept) & $0.02(0.02-0.03)^{\star}$ \\
\hline Guidelines regarding restraint (yes) & $0.60(0.49-0.74)^{\star}$ \\
\hline Refresher course regarding restraints (yes) & $0.76(0.65-0.90) *$ \\
\hline Age in years & $1.01(1.00-1.01)^{\star}$ \\
\hline Female gender & $0.76(0.69-0.83)^{\star}$ \\
\hline Number of days since admission to hospital & $1.00(1.00-1.00)^{\star}$ \\
\hline \multicolumn{2}{|l|}{$\geq 70$ completely independent } \\
\hline$\geq 60-69$ to a great extent independent & $3.18(2.73-3.70)^{\star}$ \\
\hline$\geq 45-59$ partially dependent & $8.85(7.61-10.29)^{*}$ \\
\hline$\geq 25-44$ to a great extent dependent & $24.05(20.40-28.35)^{*}$ \\
\hline$\leq 24$ completely dependent & $39.64(32.66-48.12) *$ \\
\hline Mental and behavioural disorders & $2.31(2.09-2.56)^{\star}$ \\
\hline Factors influencing health status and contact with health services & $1.41(1.21-1.64)^{*}$ \\
\hline Diseases of the digestive system & $0.83(0.75-0.93)^{\star}$ \\
\hline Diseases of the musculoskeletal system and connective tissue & $0.78(0.70-0.87)^{\star}$ \\
\hline \multicolumn{2}{|l|}{ *statistically significant based on the $95 \% \mathrm{Cl}$} \\
\hline \multicolumn{2}{|l|}{$\begin{array}{l}\text { AIC = Akaike information criterion, } I C C=\text { intraclass correlation coefficient, } \\
\text { OR }=\text { odds ratio, } 95 \% \mathrm{CI}=95 \% \text { confidence interval }\end{array}$} \\
\hline
\end{tabular}

\section{Discussion}

In this secondary data analysis of cross-sectional data on restraint use in Swiss and Austrian hospitals, we analysed the impact of organisational factors on the use of restraints in the somatic acute care hospital setting, as well as the practice 
variation among hospitals. Overall, the restraint prevalence rate was $8.7 \%$. We found that the availability of guidelines regarding restraint use on the institutional level and refresher courses for at least $80 \%$ of ward nursing staff in the last two years are associated with less restraint use. On the contrary, no association was found for the availability of a multidisciplinary expert committee regrading restraint use within the institution and regular audits on the ward level to ensure compliance with the guidelines regarding restraint use. Furthermore, the findings show that a relevant part of the variance in restraint use is explained at the hospital level (random effect), suggesting that a hospital effect exists regarding restraint use. The difference between hospitals also appears to be greater than that between countries, as might have been expected given the much higher restraint prevalence rate in Switzerland (the country variable was not selected for the model). Thus, there is evidence that, in similar patient situations, restraints are used more frequently in some hospitals than in others. This finding supports assumptions from the literature that, regarding restraint use, local habits, routine and institutional culture seem to play an important role $(18-21,42)$. Such routine or habitual restraint use, independent of an objective and evidencebased evaluation, violates professional values and fundamental human rights. Therefore, critical interprofessional reflections on the current restrictive practice within hospitals are needed to minimise non-professional, non-legal and nonethical restraint use. However, based on well-known safety models, like the Swiss cheese model, we know that patient safety is not only influenced by health professionals involved in direct patient care (micro level) (43). The conditions within an institution (meso level) and on a national level (macro level) also have a significant impact on patient safety. For this reason, critical reflection on current restraint practices should take place on micro, meso and macro level.

On the micro level, a critical interprofessional reflection of practice is only possible with appropriate knowledge about the topic of interest. Regarding restraint use, it is widely discussed that health professionals in the hospital setting do not have sufficient knowledge and expertise (21). As a result, restraints are often applied in situations that are not appropriate (14, 19, $22,44)$. For example, restraints are used for fall prevention, even though there is growing evidence that restraints are ineffective in preventing falls $(16,17)$. Also, in this study, indications could be found that knowledge influences the use of restraints, since attending a refresher course is associated with less restraint use. Thus, in line with the recommendations of a Cochrane review regarding restraint reduction in general hospitals (45), education of health professionals seems to be a relevant component for restraint reduction. In this regard, it seems important that an interprofessional approach is taken, as this is the only way to change the institutional culture, the perception of risk-taking and the work ethic (42). In particular, the results of this study show how important these institution-specific aspects seem to be (hospital effect).

However, changes in these institution-specific aspects also require a strong commitment from the meso level. First of all, there is a need for open discussion within an institution, for example to clarify responsibilities for safety (42). Especially in the care of elderly people, the assessment of security issues needs different perspectives (46). For example, functional needs must also be weighed in the decision-making process in terms of using or not using restraints. This is even more important as, like the findings show, older and more care-dependent patients have an increased risk of being restrained during their hospital stay, and as restraint use is associated with functional decline. In addition, mental and behavioural disorders are associated with a higher use of restraints. This means that a very vulnerable patient group is most affected, i.e. patients who often cannot stand up for themselves; therefore, ethical considerations are even more important. In this regard, the management has the responsibility to support front-line staff by influencing the structural conditions for example, as also shown in this study, by providing policies/ guidelines that support decision-making or at least restraint management in line with legal and ethical requirements $(18,20-22,24,45)$. In addition, they can adapt the infrastructural conditions, for example by removing restraint equipment from the wards, as it is known that the availability of restraint equipment influences its use (23). It seems interesting that, in this study, regular audits and the availability of an expert committee were not found to be associated with restraint use. A possible explanation might be that, for both tasks, the individual person (who conducts the audit or is a member of the expert committee) must be able to critically reflect on the situation in which restraints are used and, in particular, to take an outside perspective in order to identify restraint use due to the institutional culture or attitudes. However, as discussed above, the knowledge and expertise of the individual person might be insufficient and therefore no effect of these two organisational factors could be measured. 
To support critical reflection on the micro and meso level and thus to support the change in restrictive practice in order to protect human rights of personal freedom and to ensure professional restraint use, interventions should also be taken on the national (macro) level $(43,47,48)$. For example, in both included countries (Switzerland and Austria), clear legal regulations regarding restraint use in the hospital setting are lacking (15). However, clear regulations, professional statements of nurses or medical associations and national guidelines would help institutions to clarify their policies, would support the uniform education of health professionals and would provide a basis for national quality improvement programs in the hospital setting. Such programs often lead to more uniform monitoring of restraint use within institutions and thus enable comparison, which are both important aspects in restraint reduction $(24,49)$.

As restraint use is a very sensitive issue, in this respect, a national quality measurement with a risk-adjusted comparison should be considered. This is the only way to guarantee that the different patient mix of institutions is taken into account and that a fair statistical comparison can be made (43). Moreover, there is otherwise a risk that institutions with a higher restraint prevalence rate will only see their patient mix (e.g. older, more care-dependent) as the reason for the higher rate and will then reflect on the institution-specific aspects insufficiently. However, as described, this critical reflection seems to be essential for less restrictive practice. In addition, such efforts on the national level could stimulate a more open information policy regarding restraint use in hospitals, more critical thinking about restrictive practice in general and open discussions both within institutions but also in society. These aspects are well-known from similar approaches in the mental health or long-term care setting $(50,51)$.

\section{Limitations}

Beside its relevant findings, this study has some limitations. First of all, some organisational factors expected to be associated with restraint use (e.g. nurse to patient ratio) and health professional-related factors were not assessed with LPZ 2.0. It is, therefore, possible that the impact of the included organisational factors is over- or underestimated as is the relevance of the hospital effect. Secondly, it is possible that a selection bias exists. Patients who could not give informed consent and had no legal representative available had to be excluded. It could be that these patients were at high risk for restraint use and, therefore, the prevalence rate might be underestimated. Also, the impact of the predictors might be slightly different when including these patients in the analysis. Similar consequences could also be caused by a potential recall or documentation bias because restraint use was assessed over a period of 30 days. However, it is known that, regarding restraint use, the documentation is often incomplete $(5,15)$. Thirdly, the cross-sectional design has its limitations; on the one hand, the patient situations under investigation can fluctuate strongly within institutions on the measurement day and, on the other hand, no causal correlations can be identified using a cross-section design. For example, greater care dependency could lead to restraint use, but could also be a consequence of restraint use.

Despite these limitations, the results are expected to be generalisable due to the sample size and the methodological accuracy. They provide important indications for future quality development efforts. In this context, it seems to be of interest to investigate explanations for the additional $31 \%$ of explained variance on the hospital level. The inclusion of further structural characteristics in data collection and a subsequent analysis or a qualitative approach, for example by observing the (interprofessional) processes surrounding restraint use, could be helpful in this regard.

\section{Conclusions}

Regarding restraint use, a hospital effect exists. This indicates that restraints are used more frequently in certain hospitals than in others, even when considering the different patient mix. To provide restraint-free care as much as possible requires both specific knowledge and appropriate structures. Based on these findings, considerable potential for restraint reduction appears to exist in the interprofessional critical reflection of decision-making processes within a hospital; especially, the identification of situations in which restraints were used out of routine or institutional culture. This critical reflection ideally 
goes along with addressing the knowledge and attitudes towards restraints of the interprofessional team as well as of the management. A clear national (legal) regulation regarding restraint use could support a change in practice.

\section{List Of Abbreviations}

\section{LPZ}

Landelijke Prevalentiemeting Zorgkwaliteit

ICD-10

to International Statistical Classification of Diseases and Related Health Problems 10th Revision

CDS

Care Dependency Scale

$95 \% \mathrm{Cl}$

95\% confidence intervals

IQR

Interquartile range

AIC

Akaike information criterion

ICC

Intraclass correlation coefficient

VIF

Variance inflation factor

OR

Odds ratio

\section{Declarations}

\section{Ethics approval and consent to participate}

As the study was conducted in two countries, there is one ethical approval per country. In Switzerland, the Ethics Committee of the Canton of Bern declared that the present study is not subject to the Swiss Human Research Act and ethical approval was not required (April 2019, BASEC-Nr: Req-2019-00259). In Austria, the Ethics Committee of the Medical University of Graz approved the study protocol (approval nr. 20-192 ex08/09). All patients or their legal representatives received written information about the measurement and gave their verbal (Switzerland) or written (Austria) informed consent. In Switzerland, only verbal and no written consent was required, since in 2012 Swissethics and the cantonal ethics committees classified the annual LPZ data collection as a quality measurement for which no written consent of the patients is required. The decisive factors were the aim of the data collection (ensuring and further developing the quality of care), the data collection method, the type of data collected (only data of the regular care process) and the fact that no intervention is carried out. The documentation of the verbal consent was in the responsibility of the participating hospitals. It was recommended that consent be recorded in the patient documentation or centrally for all patients in a separate document. Data were collected pseudonymously so that no conclusions can be made regarding the individual patients. Participation was voluntary. The whole study was performed in accordance with the Declaration of Helsinki.

\section{Consent for publication}

Not applicable.

\section{Availability of data and materials}


The data that support the findings of this study are available from Swiss National Association for Quality Development in Hospitals and Clinics (Swiss data) and Department of Nursing Science from the Medical University of Graz (Austrian data) but restrictions apply to the availability of these data, which were used under license for the current study, and so are not publicly available.

\section{Competing interests}

The authors declare that they have no competing interests.

\section{Funding}

The quality measurement (data collection) in Switzerland was funded by the Swiss National Association for Quality Development in Hospitals and Clinics (ANQ) and in Austria by the participating hospitals themselves. The entire data analysis was financed by Bern University of Applied Sciences.

\section{Authors' contributions}

ST contributed to the conceptualization, methodology, data collection, data curation, data analysis, data interpretation and writing of the manuscript. SH supervised the project and contributed to the conceptualization, acquisition, data interpretation as well as reviewing and editing of the manuscript. SB contributed to the data collection, data interpretation and reviewing and editing of the manuscript. DR contributed to the methodology and the validation of the data analysis, the data interpretation as well as reviewing and editing of the manuscript. SZ supervised the project and contributed to the conceptualization, data interpretation as well as reviewing and editing of the manuscript. All authors read and approved the final manuscript.

\section{Acknowledgements}

We would like to thank Christa Lohrmann from the Medical University of Graz and the Swiss National Association for Quality Development in Hospitals and Clinics (ANQ) for their support with data collection. We would also like to thank Dr Reto Bürgin for sharing his expertise in multilevel analysis. Furthermore, we wish to thank all hospitals and patients for their participation in the quality measurement.

\section{Authors' information (optional)}

Not applicable.

\section{References}

1. Chou MY, Hsu YH, Wang YC, Chu CS, Liao MC, Liang CK, et al. The adverse effects of physical restraint use among older adult patients admitted to the internal medicine wards: a hospital-based retrospective cohort study. J Nutr Health Aging. 2020;24(2):160-5. https://doi.org/10.1007/s12603-019-1306-7.

2. Cusack P, Cusack FP, McAndrew S, McKeown M, Duxbury J. An integrative review exploring the physical and psychological harm inherent in using restraint in mental health inpatient settings. Int $\mathrm{J}$ Ment Health Nurs. 2018;27(3):1162-76. https://doi.org/10.1111/inm.12432.

3. Hofmann H, Hahn S. Characteristics of nursing home residents and physical restraint: a systematic literature review. J Clin Nurs. 2014;23(21-22):3012-24. https://doi.org/10.1111/jocn.12384.

4. Said AA, Kautz DD. Reducing restraint use for older adults in acute care. Nursing. 2013;43(12):59-61. https://doi.org/10.1097/01.NURSE.0000437484.75992.ca.

5. Perez D, Peters K, Wilkes L, Murphy G. Physical restraints in intensive care-An integrative review. Austral Crit Care. 2019;32(2):165-74. https://doi.org/10.1016/j.aucc.2017.12.089. 
6. W. Haugom E, Ruud T, Hynnekleiv T. Ethical challenges of seclusion in psychiatric inpatient wards: a qualitative study of the experiences of Norwegian mental health professionals. BMC Health Serv Res. 2019;19(1):879. https://doi.org/10.1186/s12913-019-4727-4.

7. Registered Nurses' Association of Ontario. Promoting Safety: Alternative Approaches to the Use of Restraints2012. https://rnao.ca/bpg/guidelines/promoting-safety-alternative-approaches-use-restraints.

8. Lachance C, Wright M-D. Avoidance of Physical Restraint Use among Hospitalized Older Adults: A Review of Clinical Effectiveness and Guidelines2019. https://www.cadth.ca/sites/default/files/pdf/htis/2019/RC1079\%20Restraint\%20Use\%20and\%20Seniors\%20Final.pdf.

9. Australian Government Aged Care Quality and Safety Commission. Minimising the use of restraints. https://www.agedcarequality.gov.au/providers/assessment-processes/minimising-restraints. Accessed 2020/09/30.

10. Australian Government Department of Health. Minimising inappropriate use of restraint in aged care. https://www.health.gov.au/initiatives-and-programs/minimising-inappropriate-use-of-restraint-in-aged-care. Accessed 2020/09/30.

11. Lombardo C, Van Bortel T, Wagner AP, Kaminskiy E, Wilson C, Krishnamoorthy T, et al. PROGRESS: the PROMISE governance framework to decrease coercion in mental healthcare. BMJ Open Qual. 2018;7(3):e000332. https://doi.org/10.1136/bmjoq-2018-000332.

12. Benbenbishty J, Adam S, Endacott R. Physical restraint use in intensive care units across Europe: The PRICE study. Intensive Crit Care Nurs. 2010;26(5):241-5. https://doi.org/10.1016/j.iccn.2010.08.003.

13. Gu T, Wang X, Deng N, Weng W. Investigating influencing factors of physical restraint use in China intensive care units: A prospective, cross-sectional, observational study. Austral Crit Care. 2019;32(3):193-8. https://doi.org/10.1016/j.aucc.2018.05.002.

14. Farina-Lopez E, Estevez-Guerra GJ, Gandoy-Crego M, Polo-Luque LM, Gomez-Cantorna C, Capezuti EA. Perception of Spanish nursing staff on the use of physical restraints. J Nurs Schol. 2014;46(5):322-30. https://doi.org/10.1111/jnu.12087.

15. Thomann S, Zwakhalen S, Richter D, Bauer S, Hahn S. Restraint use in the acute-care hospital setting: a cross-sectional multi-centre study. Int J Nurs Stud. 2021;114 https://doi.org/10.1016/j.ijnurstu.2020.103807.

16. Enns E, Rhemtulla R, Ewa V, Fruetel K, Holroyd-Leduc JM. A controlled quality improvement trial to reduce the use of physical restraints in older hospitalized adults. J Am Geriatr Soc. 2014;62(3):541-5. https://doi.org/10.1111/jgs.12710.

17. LeLaurin JH, Shorr RI. Preventing falls in hospitalized patients: state of the science. Clin Geriatr Med. 2019;35(2):273 83. https://doi.org/10.1016/j.cger.2019.01.007.

18. Goethals S, Dierckx de Casterlé B, Gastmans C. Nurses' decision-making process in cases of physical restraint in acute elderly care: A qualitative study. Int J Nurs Stud. 2013;50(5):603-12. https://doi.org/10.1016/j.ijnurstu.2012.10.006.

19. Möhler R, Meyer G. Attitudes of nurses towards the use of physical restraints in geriatric care: A systematic review of qualitative and quantitative studies. Int J Nurs Stud. 2014;51(2):274-88. https://doi.org/10.1016/j.ijnurstu.2013.10.004.

20. Perren A, Corbella D, lapichino E, Di Bernardo V, Leonardi A, Di Nicolantonio R, et al. Physical restraint in the ICU: does it prevent device removal? Minerva Anestesiol. 2015;81(10):1086-95.

21. Teece A, Baker J, Smith H. Identifying determinants for the application of physical or chemical restraint in the management of psychomotor agitation on the critical care unit. J Clin Nurs. 2020;29(1-2):5-19. https://doi.org/10.1111/jocn.15052.

22. De Bellis A, Mosel K, Curren D, Prendergast J, Harrington A, Muir-Cochrane E. Education on physical restraint reduction in dementia care: A review of the literature. Dementia. 2013;12(1):93-110.

https://doi.org/10.1177/1471301211421858. 
23. Hignett S, Sands G, Fray M, Xanthopoulou P, Healey F, Griffiths P. Which bed designs and patient characteristics increase bed rail use? Age Ageing. 2013;42(4):531-5. https://doi.org/10.1093/ageing/aft040.

24. Lach HW, Leach KM, Butcher HK. Evidence-based practice guideline: changing the practice of physical restraint use in acute care. J Gerontol Nurs. 2016;42(2):17-26. https://doi.org/10.3928/00989134-20160113-04.

25. Van Nie-Visser NC, Schols JMGA, Meesterberends E, Lohrmann C, Meijers JMM, Halfens RJG. An international prevalence measurement of care problems: study protocol. J Adv Nurs. 2013;69(9):e18-e29.

https://doi.org/10.1111/jan.12190.

26. LPZ. A solution for every health care institution. www.lpz-um.eu. Accessed 2020/12/18.

27. National Institute for Health and Care Excellence. Violence and aggression: short-term management in mental health, health and community settings. NICE; 2015. Report No.: NICE guideline 10.

28. World Health Organization. International Statistical Classification of Diseases and Related Health Problems 10th Revision. https://icd.who.int/browse10/2016/en\#/XIII. Accessed 2019/11/01.

29. Dijkstra A, Yönt GH, Korhan EA, Muszalik M, Kedziora-Kornatowska K, Suzuki M. The care dependency scale for measuring basic human needs: an international comparison. J Adv Nurs. 2012;68(10):2341-8. https://doi.org/10.1111/j.1365-2648.2011.05939.x.

30. Akaike H. A new look at the statistical model identification. IEEE Transaction on Automatic Control. 1974;19(6):716-23. https://doi.org/10.1109/TAC.1974.1100705.

31. Venables WN, Ripley BD. Modern Applied Statistics with S. 4 ed. New York: Springer; 2002.

32. Bates D, Maechler M, Bolker B, Walker S. Fitting linear mixed-effects models using Ime4. J Statistical Software. 2015;67(1):1-48. https://doi.org/10.18637/jss.v067.i01.

33. R Core Team. R: A language and environment for statistical computing. Vienna, Austria: R Foundation for Statistical Computing; 2020.

34. Subirana I, Sanz H, Vila J. Building Bivariate Tables: The compareGroups Package for R. J Statistical Software. 2014;57(12):1-16. https://doi.org/10.18637/jss.v057.i12.

35. Harrell FE, Dupont C. Hmisc: Harrell Miscellaneous. R package version 4.4-1. 2020.

36. Long JA. _jtools: Analysis and Presentation of Social Scientific Data_. R package version 2.0.1. 2019.

37. Bartoń K. MuMIn: Multi-Model Inference. R package version 1.43.17. 2020.

38. Lüdecke D. _sjPlot: Data Visualization for Statistics in Social Science_. R package version 2.8.4. 2020.

39. Yoshida K. tableone: Create 'Table 1' to Describe Baseline Characteristics. R package version 0.11.1. 2020.

40. Wickham H, Averick M, Bryan J, Chang W, McGowan L, François R, et al. Welcome to the tidyverse. J Open Source Software. 2019;4(43):1686. https://doi.org/10.21105/joss.01686.

41. IBM Corp. IBM SPSS Statistics for Windows, Version 25.0. Armonk, NY: IBM Corp.; Released 2017.

42. Kong E-H, Choi H, Evans LK. Staff perceptions of barriers to physical restraint-reduction in long-term care: a metasynthesis. J Clin Nurs. 2017;26(1-2):49-60. https://doi.org/10.1111/jocn.13418.

43. Busse R, Klazinga N, Panteli D, Quentin W, editors. Improving healthcare quality in Europe. Characteristics, effectiveness and implementation of different strategies. United Kingdom: World Health Organization (WHO), Organisation for Economic Co-operation and Development (OECD); 2019.

44. Eskandari F, Abdullah KL, Zainal NZ, Wong LP. Use of physical restraint: Nurses' knowledge, attitude, intention and practice and influencing factors. J Clin Nurs. 2017;26(23-24):4479-88. https://doi.org/10.1111/jocn.13778.

45. Abraham J, Hirt J, Kamm F, Möhler R. Interventions to reduce physical restraints in general hospital settings: a scoping review of components and characteristics. J Clin Nurs. 2020;29:3183-200. https://doi.org/10.1111/jocn.15381.

46. Dahlke SA, Hunter KF, Negrin K. Nursing practice with hospitalised older people: Safety and harm. Int J Older People Nurs. 2019;14(1):e12220. https://doi.org/10.1111/opn.12220.

Page $13 / 14$ 
47. Pivodic L, Smets T, Gambassi G, Kylänen M, Pasman HR, Payne S, et al. Physical restraining of nursing home residents in the last week of life: An epidemiological study in six European countries. Int J Nurs Stud. 2020;104:103511. https://doi.org/10.1016/j.ijnurstu.2019.103511.

48. Abraham J, Kupfer R, Behncke A, Berger-Höger B, Icks A, Haastert B, et al. Implementation of a multicomponent intervention to prevent physical restraints in nursing homes (IMPRINT): A pragmatic cluster randomized controlled trial. Int J Nurs Stud. 2019;96:27-34. https://doi.org/10.1016/j.ijnurstu.2019.03.017.

49. Muir-Cochrane E, Grimmer K, Gerace A, Bastiampillai T, Oster C. Prevalence of the use of chemical restraint in the management of challenging behaviours associated with adult mental health conditions: A meta-synthesis. J Psych Mental Health Nurs. 2019;27(4):425-45. https://doi.org/10.1111/jpm.12585.

50. Bell A, Gallacher N. Succeeding in sustained reduction in the use of restraint using the improvement model. BMJ Quality Improv Rep. 2016;5(1):u211050.w4430. https://doi.org/10.1136/bmjquality.u211050.w4430.

51. Bellenger EN, Ibrahim JE, Kennedy B, Bugeja L. Prevention of physical restraint use among nursing home residents in Australia: The top three recommendations from experts and stakeholders. Int J Older People Nurs. 2019;14(1):e12218. https://doi.org/10.1111/opn.12218.

\section{Figures}

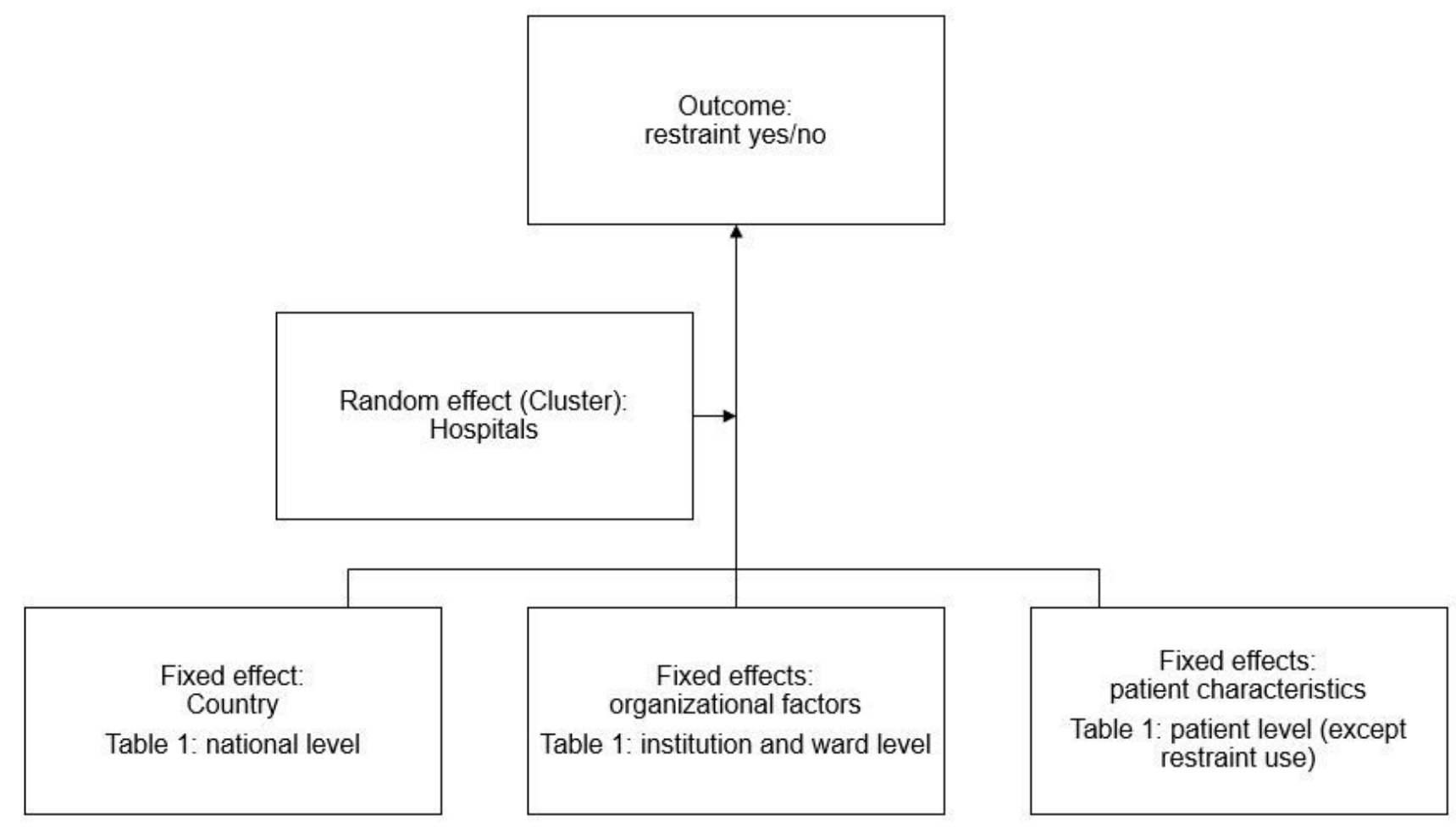

\section{Figure 1}

Baseline multilevel regression model 